\title{
Why is the Index Smile So Steep?
}

\author{
Nicole Branger
}

\author{
Christian Schlag $\ddagger$
}

This version: June 10, 2003

\footnotetext{
${ }^{\ddagger}$ Both authors are from the Faculty of Economics and Business Administration, Goethe University, Mertonstr. 17/Uni-Pf 77, D-60054 Frankfurt am Main, Germany. E-mail: branger@wiwi.uni-frankfurt.de, schlag@wiwi.uni-frankfurt.de.

Earlier versions of this paper were presented at the Brown Bag Seminar in Finance at Goethe University, the 9th Symposium on Finance, Banking, and Insurance in Karlsruhe, and the annual meetings of the Swiss Finance Association in Zurich. The authors would like to thank the seminar and conference participants for their useful comments and suggestions.
} 


\title{
Why is the Index Smile So Steep?
}

Nicole Branger

\author{
Christian Schlag
}

\begin{abstract}
Empirical evidence shows that the implied volatility smile for index options is significantly steeper than those for individual options. We propose a simple model to explain this difference. When modelling the index, an aggregation restriction has to be taken into account. The index level is a weighted sum of individual stock prices, so that the distribution of the index is completely determined by the joint distribution of the stocks. Any difference between the index smile and the smiles for individual stocks is then determined entirely by the dependence structure among the stocks. Changing this dependence structure changes the implied volatility curve for the index, leaving individual smiles unchanged.

We illustrate our idea in a jump-diffusion framework. The dependence among stocks is captured by decomposing both the jump and the diffusion terms into common and idiosyncratic parts. In a crash situation stocks are supposed to move together more than during normal market periods, which causes the difference between the implied volatilities of at-the-money and out-of-the-money puts to be much larger for the index than for individual stocks.

The relationship between the risk-neutral and the physical distribution is also of interest. It is an important feature of our model that large downward movements are caused by jumps, which behave quite differently from diffusions under a change of measure. While for purely diffusion-based models second moments are preserved under the new measure, a change of measure may alter the dependence structure of the stocks in a model with jump components.
\end{abstract}

Keywords: Option pricing, jump-diffusion, smile, implied volatility

JEL: G12, G13 


\section{Introduction and Motivation}

There is growing empirical evidence that the implied volatility smile for index options is different from that for options on individual stocks. While implied volatility is in most cases a downward sloping function of the strike price, index smiles are usually significantly steeper than the corresponding curves for individual stocks which are sometimes even flat. Among other papers this is demonstrated in Bakshi, Kapadia, and Madan (2003) for options on the S\&P 100 index, and in Bollen and Whaley (2001) for the S\&P 500. For example, Bollen and Whaley (2001) find the slope of the function relating the implied volatility of options to their moneyness to be around -0.308 for the typical stock in their sample, while for the index the curve is much steeper with a slope of -0.886 .

Two questions arise in this context. The first is how to explain in general a downward sloping volatility smile, and the second is where the differences between the implied volatility functions for indices and individual stocks come from. One hypothesis that has been put forward in the literature to explain downward sloping smiles is the so-called leverage effect. Interpreting the stock as a call option on the assets of the firm, a drop in firm value causes a drop in the stock price which increases financial leverage. This in turn should result in a higher equity volatility. However, in a recent paper Figlewski and Wang (2000) strongly reject this conjecture in an empirical study of stock options on the U.S. market. The authors do not find any significant linkage between financial leverage and volatility in a variety of tests.

Another strand of the literature explains the smile by using more advanced stochastic 
processes for the underlying asset. For example, Bakshi, Cao, and Chen (1997) present a model incorporating stochastic volatility, stochastic jumps, and stochastic interest rates. While this very general model performs better than the simple Black and Scholes (1973) model in explaining market prices for index options, it has never been tested explicitly for options on individual stocks.

In addressing the second issue Bakshi, Kapadia, and Madan (2003) argue that the different slopes of the curves are in part related to differences in the skewness of the risk-neutral distributions for the stocks and for the index. Assuming a return generating model with the market return as a factor, the difference in skewness can be explained by the properties of the idiosyncratic components. In contrast to these authors Bollen and Whaley (2001) argue that the physical distributions of the stocks and the index are not significantly different. As a consequence they try to explain the different slopes of the smiles not by the differences in the stochastic processes of the underlyings, but by buying pressure for certain out-of-the-money (OTM) index puts which serve as an insurance device against market crashes. This buying pressure increases option prices and therefore also the implied volatilities for low strike prices.

The objective of our paper is not to develop yet another derivative pricing model and to test empirically whether such a new approach is able to explain the empirical phenomena described above. We rather want to propose a simple setup which is built on well-accepted theoretical models and which offers a straightforward economic explanation for the pricing differences between equity and index options. In this sense our approach is similar in spirit to Berk (1995) who offers a simple economic rationale for the seemingly 
anomalous empirical relationship between expected stock returns and firm size.

The key feature of our approach is that it explicitly takes an additivity restriction for the index level, given individual stock prices, into account. Our model is able to generate downward sloping smiles for both options on individual stocks and on the index, as well as a significantly steeper slope of the implied volatility curve for index options. We show that these goals can be achieved without resorting to extremely advanced stochastic models and without dropping the assumption of perfect markets.

The main contribution of the paper is threefold. We first argue that differences in smiles must be explained by differences in risk-neutral distributions, not by differences in physical distributions. Indeed, in an incomplete market, the physical densities may well be the same for two assets while the risk-neutral densities are much different. This allows us to focus mainly on the distributions under the risk-neutral measure. Second, we explicitly take into account the economic aggregation restriction between an index and its component stocks. As the index level is just the weighted sum of the individual stock prices, its density is completely determined by the joint probability distribution of the individual stocks. Especially, we do not model the index as an exogenous factor explaining the returns on the stocks, but we derive the behaviour of the index from the joint behaviour of the stocks. We show that the relation between the index smile and the stock smiles is entirely determined by the structure of this dependence. Changing this dependence structure can change the index smile dramatically, although the smiles for individual stocks are left unchanged. Third, we are especially interested in the behaviour of the stocks and the index in the case of large downward price moves. The greater the 
risk-neutral probability for such a large price drop the steeper the resulting smile. Due to the aggregation restriction, a crash in the index can only occur if there is a downward move in the majority of the stocks, an issue which has been addressed in a different context by Nietert (1997). Therefore, the risk-neutral probability of a market crash depends critically on the risk-neutral probability that a downward move occurs for a large number of stocks simultaneously. This, however, implies that the slope of the index smile for low strike prices is determined by the dependence among stocks during a downward move. Indeed, there is empirical evidence that during a crash the stocks tend to move together more than during normal market periods. For example, Ang and Chen (2002) show that the dependence between stock returns and index returns during a downside move is greater than it would be under a multivariate normal distribution. Longin and Solnik (2001) as well as Campbell, Koedijk, and Kofman (2002) show that for international markets correlations increase in bear markets.

In our model we assume that each individual stock follows a jump-diffusion process with a rather simple specification. The option pricing formula for this framework is well-known and was developed by Merton (1976). In order to capture the dependence, we decompose both the diffusion and the jump term into a common and an idiosyncratic component. This decomposition can be done under the physical as well as under the riskneutral measure. It is important to note that the change of measure has quite different effects on the diffusion and the jump components. For diffusion processes neither volatilities nor correlations will change compared to the physical measure. For a jump process on the other hand the change of measure will affect variances as well as correlations. There- 
fore, the dependence structure during a crash can be very different under the physical and under the risk-neutral measure.

From an economic point of view, it can be argued that the dependence is higher under the risk-neutral measure. A simultaneous crash in all stocks will reduce the wealth of the representative investor by much more than a downward move in only a few stocks which can partially be diversified away. The fear of jumps induces investors to demand a much higher risk premium for market crashes than for downward moves in only one or a small number of stocks. In our model this is captured by a risk-neutral probability of a common downward move which is much higher than the probability of the same event under the physical measure. This setup generates downward sloping volatility curves for individual stocks as well as for the index which can exhibit different slopes. The issue of physical versus risk-neutral distributions is also discussed theoretically by Bates (2001) who relates their differences to investors' risk aversion and to potential heterogeneity between investors.

The remainder of the paper is organized as follows. In section 2 we discuss the aggregation restriction on the index. The model for individual stocks is introduced in section 3, where we also show how to incorporate the dependence structure into the model. The implications of our setup and the impact of the dependence structure on the slope of the index smile are shown in section 4. Although all our pricing arguments are of course based on risk-neutral distributions we find it nevertheless important to discuss the relationship between the risk-neutral and the physical distribution, and this is done in section 5. Some concluding remarks are presented in section 6 . 


\section{The Aggregation Restriction for the Index}

In the following analysis we will consider options on individual stocks as well as options on the index composed of these stocks. Empirically, it is observed that for both types of options the smile is downward sloping, but that the smile is much steeper for index options than for individual stock options.

We propose a model setup that is able to explain both the general shape of the implied volatility curve and the differences between stock option smiles and index smiles. In particular, our model is able to explain why the index smile is much steeper than the smiles of equity options.

When comparing the pricing of equity options to the pricing of index options, an implicit restriction has to be taken into account. It is not possible to model the behaviour of the individual stocks and to choose simply a further process for the behaviour of the index. In other words, we cannot specify some type of stochastic process and fit this process separately to each individual stock and then take some other (or the same) process to describe the random evolution of the index level. Instead, the index must obey what we call the aggregation restriction, since the index level is equal to the weighted sum of the stock prices. A very general formula for the index level $I_{t}$ at time $t$ is given by

$$
I_{t}=\sum_{i=1}^{m} w_{t}^{i} S_{t}^{i},
$$

where $S_{t}^{i}$ denotes the price of stock $i$ at time $t$ and the weights $w_{t}^{i}$ are determined by the respective index formula. For example, the weights may be related to the number of shares outstanding or to the total market capitalization of a stock. In our analysis we 
will set the weights identically equal to $1 / m$ for the sake of simplicity. This assumption is not crucial, since changing the weights will have only a marginal impact on our results. Equation (1) explicitly shows that the behaviour of the index is completely determined by the joint behaviour of the stocks.

The first step in setting up a model is to choose a stochastic process for each individual stock that is able to match the smile of equity options as it is observed in real data. The second step then is to model the dependence between the stocks. Indeed, we will show that the shape of the index smile, compared to the equity smile, is almost entirely determined by this dependence.

The slope of the smile is related to the shape of the risk-neutral distribution. The more left- (right-)skewed the distribution, the greater the implied volatilities for low (high) strike prices, and the fatter the tails of the distribution, the higher the implied volatilities for both deep in-the-money and out-of-the-money options. Recent models, like Bakshi, Cao, and Chen (1997), incorporate both stochastic volatility and jumps. Both these features are able to generate risk-neutral distributions that are more left- or right-skewed than the lognormal distribution, and that have excess kurtosis. The exact effects depend on the ultimate process and the actual parameters. Among other things Das and Sundaram (1999) discuss how the effects of stochastic volatility and jumps depend on time to maturity. They show that stochastic volatility has a greater impact for longer maturities, while jumps are necessary for short-term smiles to exhibit significantly negative slopes (given that the average size of a jump is negative).

Our objective is to explain a downward sloping smile, so that the risk-neutral distri- 
bution of returns must be left-skewed. In order to achieve this for both individual stock options and the index, we rely on downward jumps for both the individual stocks and the index. The steepness of the smile curves then depends on the risk-neutral probabilities of these (large) downward moves as well as on the jump sizes. Given the jump parameters for the stocks, the jump characteristics of the index depend on the joint behaviour of the stocks due to the aggregation restriction. There can only be a crash in the index when there is a downward move in the majority of the stocks (see also Nietert (1997)). The probability of a market crash therefore depends on the probability that a downward jump in a stock is a common jump affecting more or less all of the stocks simultaneously. The greater this probability under the risk-neutral measure, the steeper the smile for index options, while individual smiles will not be affected.

To get the intuition for the importance of the dependence structure consider two extreme scenarios. In the first case, the jumps in the stocks all occur independently, i.e. the probability for a market crash is extremely low. Here we expect the impact of jumps in the individual stocks on the prices of index options to be small because of the pronounced diversification effect. In the second case, assume that downward jumps in the stocks all occur at the same time. Whenever there is a crash in one stock, the prices of all the component stocks will go down, resulting in a market crash. In this case, the jump component has a high impact on the prices of OTM index puts, and the index smile will be quite steep. 


\section{Pricing Individual Stock Options}

As discussed above the stochastic behaviour of the index can not be modelled independently of that of the stocks comprising the index. It is rather determined by the stochastic processes of the individual stocks and their dependence. Our main focus is on this joint behaviour of the stocks: How much do the stock price movements depend on each other? We use a model setup that is as simple as possible and that allows us to focus on the analysis of the dependence structure. Each individual stock follows a jump-diffusion process, where the parameters are assumed to be identical across stocks. Furthermore, the measures of dependence are assumed to be equal for any two pairs of stocks. The correlation matrix would thus have equal entries for all elements off the main diagonal. These assumptions only serve the purpose of keeping the model as simple as possible. They could be easily relaxed without a significant change in the results of the following analysis.

Each stock is driven by a Wiener process and a Poisson process, where the Poisson process obviously captures the jumps in the stock price. Both the Wiener process and the Poisson process have a common and an idiosyncratic component. The common component affects all stocks in the index simultaneously, whereas the idiosyncratic components are mutually independent. Formally for stock $i$ we assume the dynamics

$$
d S_{t}^{i}=\mu^{i} S_{t-}^{i} d t+\sigma S_{t-}^{i}\left(\sqrt{\rho_{W}} d W_{t}^{c}+\sqrt{1-\rho_{W}} d W_{t}^{i}\right)-\gamma S_{t-}^{i}\left(d N_{t}^{c}+d N_{t}^{i}\right) .
$$

Here $W^{c}$ is the Wiener process that is common to all stocks, and $W^{i}$ is the purely idiosyncratic diffusion component of asset $i$ independent of $W^{c}$. This independence property implies that $\sqrt{\rho_{W}} W^{c}+\sqrt{1-\rho_{W}} W^{i}$ is again a Wiener process for every stock $i$. Further- 
more, we assume a constant correlation of the diffusion parts between any two stocks equal to $\rho_{W} \geq 0$.

In case of a jump the stock price decreases by the constant factor $\gamma$. Jumps have intensity $h$ so that for some small $\Delta t$ the probability of a jump occuring in the interval $[t, t+\Delta t]$ is approximately equal to $h \Delta t$. A jump is a common jump with probability $\rho_{N}$ and an idiosyncratic jump with probability $1-\rho_{N}$. This is modelled by defining the common Poisson process $N^{c}$ with intensity $h \rho_{N}$ and the purely idiosyncratic Poisson process $N^{i}$ with intensity $h\left(1-\rho_{N}\right)$. The common Poisson process captures market crashes, and the greater $\rho_{N}$, the greater the probability of such a crash. $N_{t}^{c}+N_{t}^{i}$ is again a Poisson process with intensity $h$. Note that for a Poisson process $N$, the probability that there are two or more jumps in a very short interval is zero. Intuitively, this means that $d N_{t}$ can only take on the values 0 and 1 . This is also true for the term $d N_{t}^{c}+d N_{t}^{i}$ as the probability that there is a jump for each of these independent processes during a very short interval of time is again equal to zero.

The fact that all the parameters in equation (2) are assumed to be constant may seem restrictive at first. However, we are interested in isolating the truly important drivers of the differences in the slopes of the smiles between equity and index options. Therefore we focus on the simplest possible setup, with which we will nevertheless be able to explain the stylized empirical findings reported in the introduction. The qualitative results of our model should not change significantly if it was augmented by, e.g., stochastic volatility, random jump sizes, stochastic jump intensities, or stochastic correlation coefficients.

In a first step, we now consider the pricing of options on individual stocks. For 
this analysis, the distinction between idiosyncratic components and common components does not matter. Only the total risk of the stock is important, which is determined by the sum of the diffusion components and by the sum of the Poisson processes. For the purpose of pricing options on individual stocks we can therefore rewrite the dynamics from equation (2) as

$$
d S_{t}=\mu S_{t-} d t+\sigma S_{t-} d W_{t}-\gamma S_{t-} d N_{t}
$$

Here $W$ is a Wiener process, and $N$ is a Poisson process with intensity $h$. Under the risk-neutral measure the stock price process becomes

$$
d S_{t}=(r+\widehat{h} \gamma) S_{t-} d t+\sigma S_{t-} d \widehat{W}_{t}-\gamma S_{t-} d N_{t}
$$

where $\widehat{W}$ is again a Wiener process. The intensity of the Poisson process changes from $h$ to $\widehat{h}=\phi h$, where $\phi>0$ is the market price of jump risk. The variance of the continuous return is given by

$$
\widehat{\operatorname{Var}}\left[\ln S_{t}-\ln S_{0}\right]=\left(\sigma^{2}+\widehat{h}(\ln (1-\gamma))^{2}\right) t
$$

This variance depends on the jump intensity, so that it will in general be different under the physical and the risk-neutral measure. Note that this is a special feature of models incorporating jump components. For a pure diffusion model we would have $\widehat{\operatorname{Var}}\left[\ln S_{t}-\ln S_{0}\right]=\operatorname{Var}\left[\ln S_{t}-\ln S_{0}\right]$, i.e. the variance would not be affected by the measure change.

As shown by Merton (1976) the price of a European call option on the stock in the 
framework of equations (3) and (4) is given by

$$
C^{J D}\left(S_{0}, K, r, T, \sigma, \widehat{h}, \gamma\right)=\sum_{n=0}^{N} \frac{(\widehat{h}(1-\gamma) T)^{n}}{n !} e^{-\widehat{h}(1-\gamma) T} C^{B S}\left(S_{0}, K, r_{n}, T, \sigma\right)
$$

where

$$
r_{n}=r+\widehat{h} \gamma+\frac{n \ln (1-\gamma)}{T}
$$

and $C^{B S}(S, K, r, T, \sigma)$ is the price of a European call option in the Black-Scholes model.

In our model setup with $\gamma>0$, the risk-neutral distribution has a higher variance and is more left-skewed than a lognormal distribution. Therefore, the implied volatility function is in general decreasing in the strike price. The higher the jump intensity and the higher the jump size, the higher the implied volatilities for options with low strikes, and the steeper the smile.

We now return to the question of how to model the joint behaviour of the stocks under the risk-neutral measure. Under the physical measure, equation (2) shows that the joint behaviour is governed by $\rho_{W}$ and by $\rho_{N}$. Under the risk-neutral measure, the stock price process is

$$
d S_{t}^{i}=(r+\hat{h} \gamma) S_{t-}^{i} d t+\sigma S_{t-}^{i}\left(\sqrt{\rho_{W}} d \widehat{W}_{t}^{c}+\sqrt{1-\rho_{W}} d \widehat{W}_{t}^{i}\right)-\gamma S_{t-}^{i}\left(d N_{t}^{c}+d N_{t}^{i}\right)
$$

Here $\widehat{W}^{c}$ and $\widehat{W}^{i}$ are again Wiener processes. If we only consider the diffusion components, the correlation of any two stocks under the risk-neutral measure is again $\rho_{W}$. However, in analogy to (4) the intensity of the Poisson process $N^{c}+N^{i}$ changes from $h$ to $\widehat{h}=h \phi$, and also the probability that a jump is common changes from $\rho_{N}$ to $\widehat{\rho}_{N}$. As the risk-neutral measure and the physical measure must be equivalent, i.e. assign a zero probability to 
identical sets of events, $\rho_{N}=0$ implies $\widehat{\rho}_{N}=0$, and $\rho_{N}=1$ implies $\widehat{\rho}_{N}=1$. Note that this is all we can say about the relationship between $\rho_{N}$ and $\widehat{\rho}_{N}$ so that for $\rho_{N} \in(0,1)$ the only restriction is $\widehat{\rho}_{N} \in(0,1)$.

As already shown, the variance of the jump components is different under the physical and under the risk-neutral measure. Furthermore, the correlation of the jump components $N^{c}+N^{i}$ and $N^{c}+N^{j}$ of stocks $i$ and $j$ is different under the physical and under the risk-neutral measure as well. Under the physical measure,

$$
\operatorname{corr}\left[N_{t}^{c}+N_{t}^{i}, N_{t}^{c}+N_{t}^{j}\right]=\rho_{N},
$$

whereas under the risk-neutral measure

$$
\widehat{\operatorname{corr}}\left[N_{t}^{c}+N_{t}^{i}, N_{t}^{c}+N_{t}^{j}\right]=\widehat{\rho}_{N} .
$$

This change in second moments is totally different from what we obtain in a pure diffusion setting, where not only variances, but also correlations remain unchanged in the course of a measure change.

This fact may in part explain why physical distributions do not seem very helpful in explaining the shapes of smiles observed in the options markets, as documented by Bollen and Whaley (2001). Whereas in a multivariate diffusion model we can estimate the diversification effect from stock market data, this is basically impossible for models containing jumps. The lower tail can be quite different under the physical and under the risk-neutral distribution, since correlations can change almost arbitrarily. In particular, we can no longer use the dependence under the physical probability to assess the dependence under the risk-neutral distribution. 


\section{Pricing Index Options}

Having modelled the joint behaviour of the stocks, we now consider the characteristics of the index. Assuming $S_{0}^{i}=1$ for $i=1, \ldots, m$ and weights that are identically equal to $1 / m$ the index level at time $t$ is given as

$$
\begin{aligned}
I_{t}= & \frac{1}{m} \underbrace{\exp \left\{\left(r-0.5 \sigma^{2}+\gamma \widehat{h}\right) t+\left(\sqrt{\rho_{W}} \widehat{W}_{t}^{c}\right\}(1-\gamma)^{N_{t}^{c}}\right.}_{\text {common component }} \\
& \times \underbrace{\sum_{i=1}^{m} \exp \left\{\sqrt{1-\rho_{W}} \widehat{W}_{t}^{i}\right\}(1-\gamma)^{N_{t}^{i}}}_{\text {idiosyncratic components }}
\end{aligned}
$$

The first term represents the common component, since the respective impact of $\widehat{W}^{c}$ and $N^{c}$ is the same for each stock and for the index. The second term is the sum of $m$ idiosyncratic components. The lower the correlation coefficients $\rho_{W}$ and $\widehat{\rho}_{N}$, the more diversification effects will show up in this term.

In the following analysis, we are primarily interested in the influence of $\rho_{W}$ and of $\widehat{\rho}_{N}$ on the slope of the index smile. As we will show the impact of these two correlation measures on the shape of the smile for index options is distinctively different. Note further that these parameters have no impact on the prices of options on individual stocks. As argued above, these prices only depend on the total diffusion component and the total jump component, but not on the decomposition of these components into common and idiosyncratic parts. Taken together, changing the dependence between the stocks via $\rho_{W}$ and $\widehat{\rho}_{N}$ changes the smile of the index without changing the smile of individual stocks. These parameters can therefore be used to achieve a given difference between the smile of stocks and the smile of the index. 
There is no closed-form solution for index option prices in our setting. Since the index is some weighted arithmetic average of individual stock prices, such a formula would not exist even if each stock followed a geometric Brownian motion and all the stocks were independent. We therefore use Monte Carlo simulation to calculate the prices of index call options. From these, we infer the implied volatilities and compare them to the implied volatilities of the stocks.

Our base case has the following parameters (note that the parameters are identical for all the individual stocks and pairs of stocks): $S_{0}=1, r=0, T=1 / 12, \sigma=0.2$, $\gamma=0.2, \widehat{h}=1.0, \rho_{W}=0.2$, and $\widehat{\rho}_{N}=1.0$. To calculate the option prices we use $1,000,000$ simulation runs to generate the distribution of the terminal prices of $m=30$ stocks. The number of common and of idiosyncratic jumps is drawn from independent Poisson distributions with intensities $\widehat{\rho}_{N} \widehat{h} T$ and $\left(1-\widehat{\rho}_{N}\right) \widehat{h} T$, respectively. Of course, more than one jump may occur over the interval $[0, T]$.

Before we analyze our model in detail we want to make sure that the different slopes of the smiles for equity and index options are not due to the simple and well-known fact that the sum of lognormal random variables is itself not lognormally distributed (except in the extreme case of perfect correlation). In a scenario with lognormal individual stock prices the index follows a distribution which cannot be represented in closed form. As the results of extensive simulations have shown, the deviation of the index from lognormality can certainly not explain the empirically observed differences between the slopes of the smile curves (which are in this scenario, of course, flat for individual stocks). The differences are marginal, most of the time hardly visible. So we conclude that other factors 
must be responsible for the empirically observed substantial discrepancies between equity and index option smiles.

Figure 1 shows the impact of $\rho_{W}$ on the implied volatilities of index options. For low strike prices, the influence of $\rho_{W}$ on implied volatilities can almost be neglected. For high strike prices, the greater the correlation $\rho_{W}$, the greater the implied volatility for index options. Note that some of the curves fall off sharply for high strikes. This is due to the fact that our simulations hardly produce any realizations of the terminal index value in this range. Option prices for such high strikes are therefore practically equal to zero, so that implied volatilities are not well-defined.

The effect on the implied volalitities for high strike prices can be explained via the variance of the index. The greater the correlation of the individual Wiener processes, the greater the variance of the index, since there is less diversification. This increases the prices of index options, resulting in a higher implied index volatility. To get the intuition for what happens for low strike prices, note that low realisations of the index are reached either by low realisations of the Wiener process, or by downward jumps. Below a certain level the probability that a price in this range is reached by the Wiener process is nearly zero, while the probability that it is reached by downward jumps may be comparatively high so that the implied volatility for options with quite low strike prices is mainly determined by the characteristics of the jump component. This explains that implied volatilities for low strike prices are not affected significantly by the correlation $\rho_{W}$ of the Wiener processes.

The graphs in figure 2 show the implied volatility graphs for varying values of $\widehat{\rho}_{N}$, the correlation measure for the Poisson processes. Also here it is possible that some high 
index levels are just not reached at all in the simulation, so that implied volatilities do not exist for the corresponding strike prices. Compared to the previous figure the picture is exactly reversed. Again, implied volatilites are increasing in the correlation measure. However, now we observe a much larger impact for low strike price options, whereas the effect of an increase in $\widehat{\rho}_{N}$ is almost negligible for out-of-the-money calls.

Again the explanation is found by looking at the total variance of the index. As mentioned above the greater the correlation, the less diversification there is and the greater the variance will be. Therefore, the overall level of implied volatilities increases in $\widehat{\rho}_{N}$. The different influence of $\widehat{\rho}_{N}$ on options with low and high strike prices can be explained by noting that we only consider downward jumps. The greater $\widehat{\rho}_{N}$, the greater the probability that there is a crash in the index, and the greater the price of an out-of-the-money put on the index. The price of an out-of-the-money call, on the other hand, is hardly affected, because high levels of the index are not reached by jumps, but only due to large positive shocks in the diffusion part.

Taken altogether, implied volatilities for low strike prices are increasing in $\widehat{\rho}_{N}$. Implied volatilities for high strike prices are increasing in $\rho_{W}$. The absolute amount of the slope is therefore increasing in $\widehat{\rho}_{N}$ and decreasing in $\rho_{W}$. This shows that there is basically no need to introduce more complex stochastic processes for the individual stocks to generate the differences between implied volatility curves that are observed in real data. In the case of index options the dependence structure between the stocks in the index is the key variable in the model, and it is mainly this dependence that generates steeper slopes for the index, based on a model which also generates negatively sloped smiles for 
individual stocks.

\section{$5 \quad$ Linking Physical and Risk-Neutral Distributions}

For the pricing of derivative assets only the risk-neutral distribution and not the physical distribution is relevant. Nevertheless, it is interesting to take a closer look at the distribution under the original measure to see what we can and what we cannot infer from it.

In our model each stock follows a jump-diffusion process under the physical distribution. We assume that the processes are the same for each stock so that the physical distributions are the same. However, this does not at all mean that also the risk-neutral distributions have to coincide. Because there are more risk factors than traded assets, the market is incomplete. So it is very well possible that the market prices of risk for the jump components of two stocks are different, implying that the risk-neutral densities and thus also the smile curves of the two stocks will be different. Differences in the physical distributions are therefore certainly one potential source for differences in the smiles, but they are by no means the only one.

Concerning the dependence structure of the individual stocks, the picture is a little bit more complicated. We model the co-movement of the stocks by decomposing the diffusion part and the jump part into a common and an idiosyncratic component. The correlation $\rho_{W}$ of the Wiener processes describes how much diversification there is in the index for normal movements of the stocks. It is the same under the physical and the 
risk-neutral measure.

The correlation $\rho_{N}$ of the Poisson processes gives the physical probability that a jump is a common one, i.e. it determines - together with the intensity - the probability of a market crash. Under the risk-neutral measure this correlation is changed to $\widehat{\rho}_{N}$ so that the dependence of the stocks in the lower tail can be completely different under the physical than under the risk-neutral distribution. It is well possible that $\rho_{N}$ is close to zero and $\widehat{\rho}_{N}$ is close to one so that the probability of a market crash is quite low under the physical density, whereas it is substantial under the risk-neutral density.

Altogether, since the physical and the risk-neutral measure must be equivalent, the role of the physical distribution with respect to the jump component is only to specify which events are possible. It only tells us that there are common jumps and idiosyncratic jumps, and that the jump size is $-\gamma$. The risk-neutral probabilities of common and idiosyncratic jumps are not specified at all, since in our setup, the market is incomplete. One could well argue that the probability of a jump is much higher under the risk-neutral distribution due to risk-aversion - remember that the change of measure depends on the risk attitude of the investors. We can also argue that the conditional probability that a jump is a common jump causing a crash in the index is much higher under the risk-neutral measure. Again, this can be motivated by risk-aversion, since a common jump will always reduce the wealth of an investor significantly while an idiosyncratic jump could partly be diversified away. 


\section{Conclusion}

An index is a portfolio of stocks, so that the index level is a weighted sum of individual stock prices. As a consequence the stochastic behaviour of the index is completely determined by the joint behaviour of the stocks, i.e. by the individual stochastic processes of the stocks and by their dependence structure. This paper explicitly takes this aggregation restriction into account when comparing the implied volatility smiles of options on individual stocks and on the index.

We show that it is primarily the dependence structure that is able to explain the differential pricing of stock and index options. Assuming for simplicity that each jump is a downward jump, we show that the skew is the steeper the greater the correlation of the jumps and the lower the correlation of the Wiener processes.

In our analysis we highlight the fundamental differences between diffusions and jumps. For diffusions second moments are identical under the physical and under the riskneutral measure. Changing the measure does not change the dependence structure. On the contrary, for jumps second moments can be different under the physical and under the risk-neutral measure. Changing the measure can change the dependence structure completely so that we are free to choose nearly any dependence structure under the riskneutral measure, the only restriction being the equivalence to the physical measure.

Therefore, the inclusion of jump components is critical in our model. When calibrating the model to time-series and cross-sections of option prices, stock prices, and index prices, then from a conceptual point of view the time-series information gives the second 
moments of the diffusion under the physical and under the risk-neutral measure. The cross-section of stock option prices gives the characteristics of jump intensities and jump sizes under the risk-neutral measure. Finally, for calibrating the model to index options also, we can choose the correlations of the jumps and of the jump sizes. Without the inclusion of jumps, we would not have this additional degree of freedom.

Of course, our approach is not restricted at all to the simple setup we have proposed in this paper. More complicated dependence structures and more complicated models for the individual stocks can easily be introduced. Our main results, namely that the aggregation restriction must be taken into account and that the dependence structure of the stocks determines the relation between individual smiles and index smiles, do not depend on the particular model chosen for the empirical application. Concerning the dependence structure we are not limited to considering only common and idiosyncratic components, but one could also think of additional components that only affect certain subsets of the universe of all stocks. We are also not restricted to a constant correlation between any two stocks but can start from an arbitrary correlation structure. Concerning the stochastic processes of the stocks we can integrate our approach into a more complicated model setup allowing for stochastic jumps, stochastic volatility, and stochastic interest rates, like the models suggested by Bakshi, Cao, and Chen (1997) or Pan (2002). 


\section{References}

Ang, A., and J. Chen (2002) Asymmetric Correlations of Equity Portfolios, Journal of Financial Economics 63, 443-494.

Bakshi, G., C. Cao, and Z. Chen (1997) Empirical Performance of Alternative Option Pricing Models, Journal of Finance 52, 2003-2049.

Bakshi, G., N. Kapadia, and D. Madan (2003) Stock Return Characteristics, Skew Laws, and the Differential Pricing of Individual Equity Options, Review of Financial Studies 16, 101-143.

Bates, D. S. (2001) The Market for Crash Risk, Working Paper.

Berk, J. (1995) A Critique of Size-Related Anomalies, Review of Financial Studies 8, $275-286$.

Black, F., and M. Scholes (1973) The Pricing of Options and Corporate Liabilities, Journal of Political Economy 81, 637-654.

Bollen, N. P. P., and R. E. Whaley (2001) What Determines the Shape of Implied Volatility Functions?, Working Paper.

Campbell, R., K. Koedijk, and P. Kofman (2002) Increased Correlation in Bear Markets: A Downside Risk Perspective, Financial Analysts Journal 58.

Das, S. R., and R. K. Sundaram (1999) Of Smiles and Smirks: A Term Structure Perspective, Journal of Financial and Quantitative Analysis 34, 211-240. 
Figlewski, S., and X. Wang (2000) Is the "Leverage Effect" a Leverage Effect?, Working Paper.

Longin, F., and B. Solnik (2001) Extreme Correlations of International Equity Markets, Journal of Finance 61, 649-676.

Merton, R. C. (1976) Option Pricing When Underlying Stock Returns are Discontinuous, Journal of Financial Economics 3, 125-144.

Nietert, B. (1997) Jump/Diffusion Option Pricing - a Reexamination from an Economic Viewpoint, Working Paper 3/1997.

Pan, J. (2002) The Jump-Risk Premia Implicit in Options: Evidence from an Integrated Time-Series Study, Journal of Financial Economics 63, 3-50. 


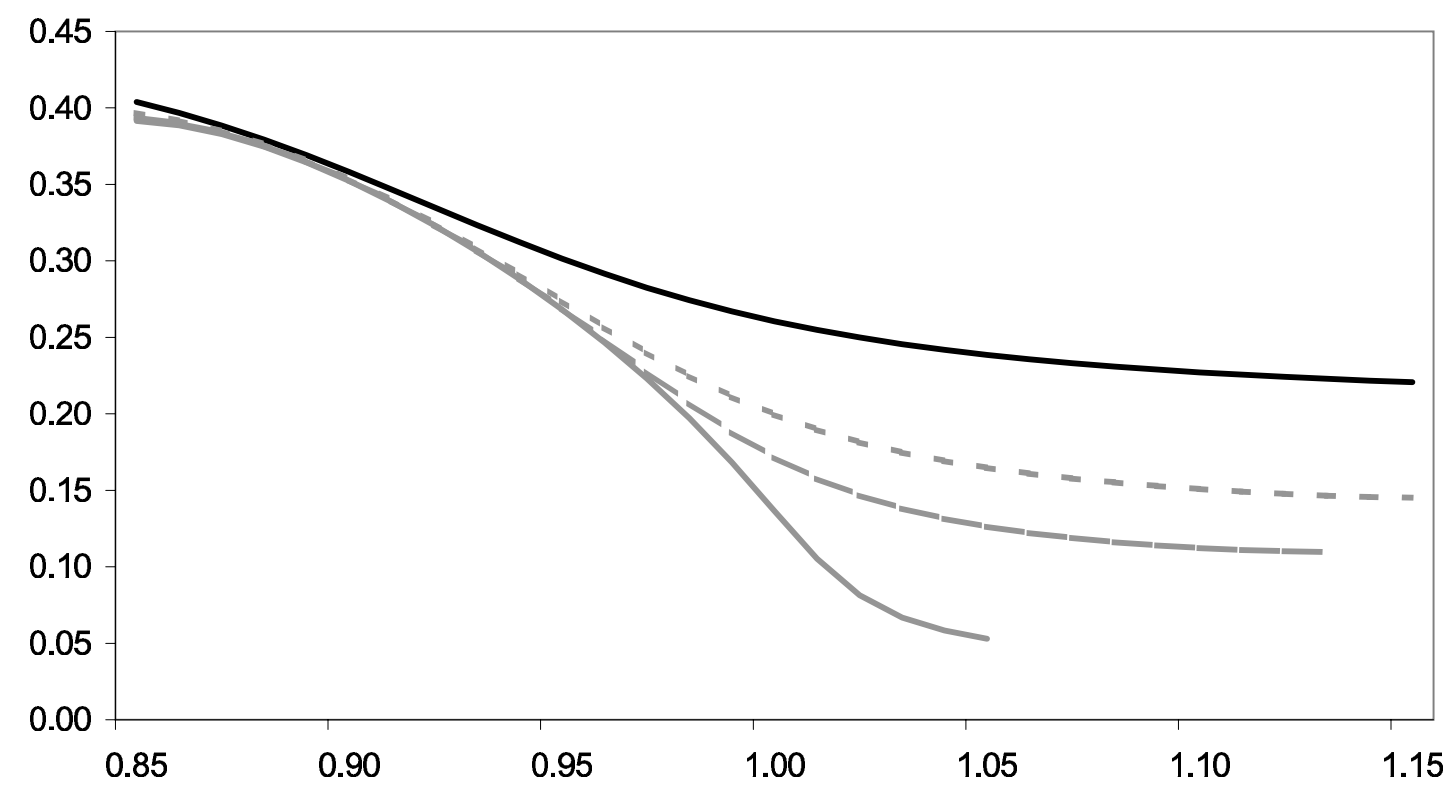

Figure 1:

Jump-diffusion model

Implied volatility curves for the representative stock (upper line) and for the index as a function of strike price for different values of $\rho_{W}$

(from bottom to top, $\rho_{W}=0.00,0.20,0.40$ )

$S_{0}=1, r=0.00, T=1 / 12, \sigma=0.20, \widehat{h}=1.00, \gamma=0.2, \widehat{\rho}_{N}=1.00$ 


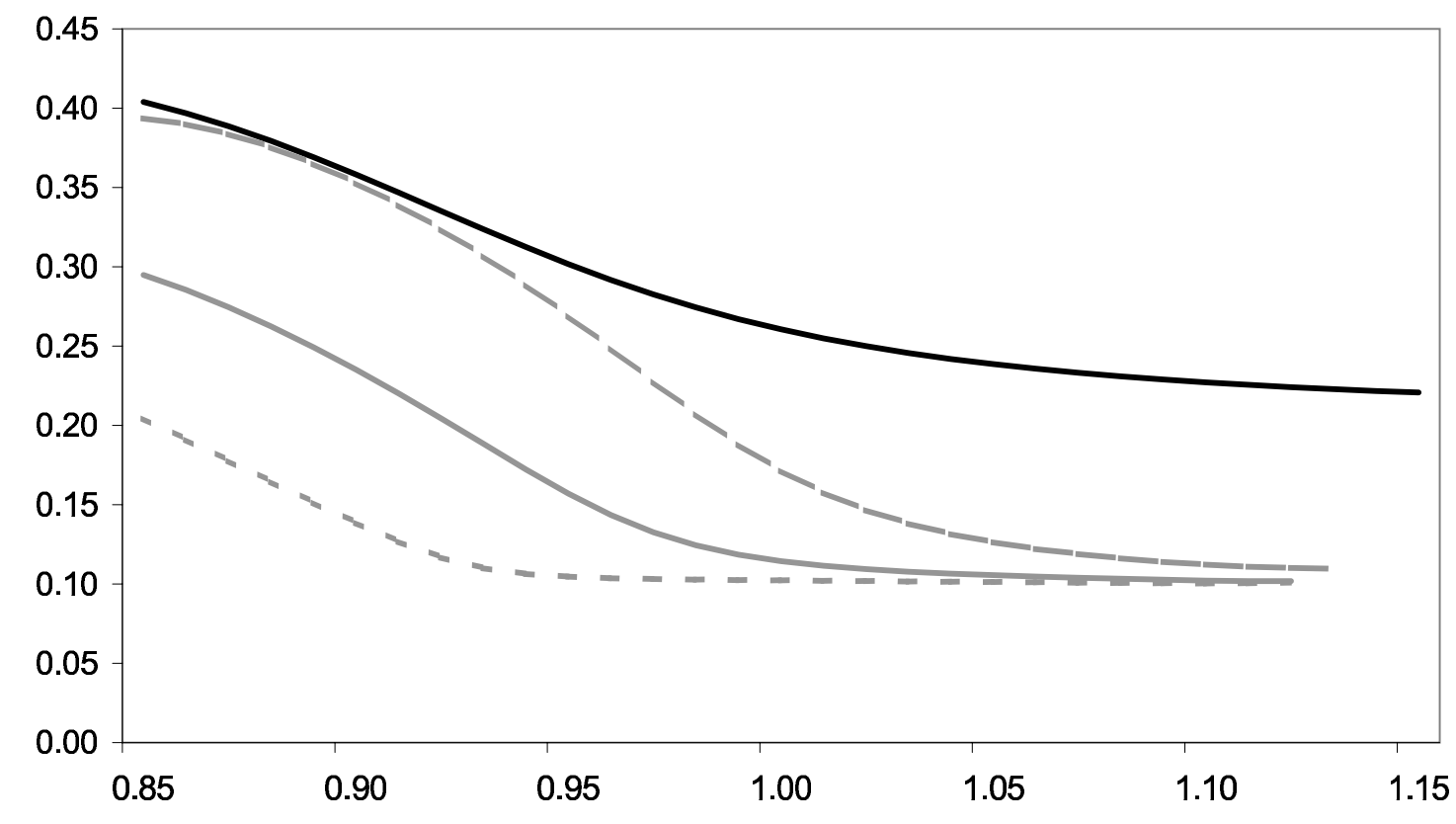

Figure 2:

Jump-diffusion model

Implied volatility curves for the representative stock (upper line) and for the index as a function of strike price for different values of $\widehat{\rho}_{N}$

(from bottom to top, $\widehat{\rho}_{N}=0.00,0.20,1.00$ )

$S_{0}=1, r=0.00, T=1 / 12, \sigma=0.20, \rho_{W}=0.20, \widehat{h}=1.00, \gamma=0.2$ 